\title{
New oscillation criteria for higher order delay dynamic equations on time scales
}

\author{
Taixiang Sun ${ }^{1 *}$, Weiyong $\mathrm{Yu}^{2}$ and Qiuli $\mathrm{He}^{3}$
}

\section{"Correspondence:}

stx1963@163.com

${ }^{1}$ Guangxi Colleges and Universities

Key Laboratory of Mathematics and

Its Applications, Nanning, Guangxi 530004, P.R. China

Full list of author information is

available at the end of the article

\begin{abstract}
In this paper, we investigate the oscillation of the following higher order delay dynamic equation: $\left\{a_{n}(t)\left[\left(a_{n-1}(t)\left(\cdots\left(a_{1}(t) x^{\Delta}(t)\right)^{\Delta} \cdots\right)^{\Delta}\right)^{\Delta}\right]^{\alpha}\right\}^{\Delta}+g(t, x(\tau(t)))=0$ on any time scale $\mathbf{T}$ with $\sup \mathbf{T}=\infty$. Here $n \geq 2, a_{k}(t) \in C_{\mathrm{rd}}(\mathbf{T},(0, \infty))(1 \leq k \leq n), \tau: \mathbf{T} \rightarrow \mathbf{T}$ is an increasing differentiable function with $\tau(t) \leq t$ and $\lim _{t \rightarrow \infty} \tau(t)=\infty$, $g \in C(\mathbf{T} \times \mathbf{R}, \mathbf{R})$ with $g(t, x) / x^{\beta} \geq q(t)$ for some $q(t) \in C_{\mathrm{rd}}(\mathbf{T},(0, \infty))$ when $x \neq 0$, and $\alpha \geq 1, \beta \geq 1$ are two quotients of odd positive integers. We give sufficient conditions under which every solution of this equation is either oscillatory or tends to zero.

MSC: $34 \mathrm{~K} 11 ; 34 \mathrm{~N} 05 ; 39 \mathrm{~A} 10$

Keywords: oscillation; dynamic equation; time scale
\end{abstract}

\section{Introduction}

In this paper, we investigate the oscillation of the following higher order delay dynamic equation:

$$
\left\{a_{n}(t)\left[\left(a_{n-1}(t)\left(\cdots\left(a_{1}(t) x^{\Delta}(t)\right)^{\Delta} \cdots\right)^{\Delta}\right)^{\Delta}\right]^{\alpha}\right\}^{\Delta}+g(t, x(\tau(t)))=0
$$

on some time scale $\mathbf{T}$. Here $n \geq 2, a_{k}(t) \in C_{\mathrm{rd}}(\mathbf{T},(0, \infty))(1 \leq k \leq n), \tau: \mathbf{T} \rightarrow \mathbf{T}$ is an increasing differentiable function with $\tau(t) \leq t$ and $\lim _{t \rightarrow \infty} \tau(t)=\infty, g \in C(\mathbf{T} \times \mathbf{R}, \mathbf{R})$ with $g(t, x) / x^{\beta} \geq q(t)$ for some $q(t) \in C_{\mathrm{rd}}(\mathbf{T},(0, \infty))$ when $x \neq 0$, and $\alpha \geq 1, \beta \geq 1$ are two quotients of odd positive integers. Write

$$
S_{k}(t, x(t))= \begin{cases}x(t), & \text { if } k=0, \\ a_{k}(t) S_{k-1}^{\Delta}(t, x(t)), & \text { if } 1 \leq k \leq n-1, \\ a_{n}(t)\left[S_{n-1}^{\Delta}(t, x(t))\right]^{\alpha}, & \text { if } k=n,\end{cases}
$$

then $(E)$ reduces to the equation

$$
S_{n}^{\Delta}(t, x(t))+g(t, x(\tau(t)))=0
$$

Since we are interested in the oscillatory behavior of solutions near infinity, we assume that $\sup \mathbf{T}=\infty$ and $t_{0} \in \mathbf{T}$ is a constant. We define the time scale interval $[a, \infty)_{\mathbf{T}}=$ $\{t \in \mathbf{T}: t \geq a\}$. A nontrivial real-valued function $x$ is said to be a solution of (1.1) if $x \in C_{\mathrm{rd}}\left(\left[T_{x}, \infty\right)_{\mathrm{T}}, \mathbf{R}\right), T_{x} \geq t_{0}$, which has the property that $S_{k}(t, x) \in C_{\mathrm{rd}}^{1}\left(\left[T_{x}, \infty\right)_{\mathrm{T}}, \mathbf{R}\right)$ for

\section{Springer}

○2014 Sun et al.; licensee Springer. This is an Open Access article distributed under the terms of the Creative Commons Attribution License (http://creativecommons.org/licenses/by/2.0), which permits unrestricted use, distribution, and reproduction in any medium, provided the original work is properly cited. 
$1 \leq k \leq n$, and satisfies $(1.1)$ on $\left[T_{x}, \infty\right)_{\mathrm{T}}$. The solutions vanishing in some neighborhood of infinity will be excluded from our consideration. A solution $x$ of (1.1) is said to be oscillatory if it is neither eventually positive nor eventually negative, otherwise it is called nonoscillatory. The theory of time scales, which has recently received a lot of attention, was introduced by Stefan Hilger in [1] in order to unify continuous and discrete analysis. The cases when a time scale $\mathbf{T}$ is equal to $\mathbf{R}$ or the set of all integers $\mathbf{Z}$ represent the classical theories of differential and difference equations. Many results concerning differential equations carry over quite easily to corresponding results for difference equations, while other results seem to be completely different from their continuous counterparts. The study of dynamic equations on time scales reveals such discrepancies, and it helps avoid proving results twice-once for differential equations and once again for difference equations. The general is to prove a result for a dynamic equation where the domain of the unknown function is a time scale $\mathbf{T}$. In this way results not only related to the set of real numbers or set of integers but those pertaining to more general time scales are obtained. Therefore, not only can the theory of dynamic equations unify the theories of differential equations and difference equations, but it also extends these classical cases to cases 'in between', e.g., to the so-called $q$-difference equations when $\mathbf{T}=\left\{1, q, q^{2}, \ldots, q^{n}, \ldots\right\}$, which has important applications in quantum theory (see [2]). In this work, knowledge and understanding of time scales and time scale notation are assumed, for an excellent introduction to the calculus on time scales; see Bohner and Peterson [3, 4]. In recent years, there has been much research activity concerning the oscillation and asymptotic behavior of solutions of some dynamic equations on time scales.

In [5], Hassan studied the third-order dynamic equation

$$
\left(a(t)\left\{\left[r(t) x^{\Delta}(t)\right]^{\Delta}\right\}^{\gamma}\right)^{\Delta}+f(t, x(\tau(t)))=0
$$

on a time scale $\mathbf{T}$, where $\gamma \geq 1$ is the quotient of odd positive integers, $a$ and $r$ are positive rd-continuous functions on $\mathbf{T}$, and the so-called delay function $\tau: \mathbf{T} \rightarrow \mathbf{T}$ satisfies $\tau(t) \leq t$ for $t \in \mathbf{T}$ and $\lim _{t \rightarrow \infty} \tau(t)=\infty$ and $f \in C(\mathbf{T} \times \mathbf{R}, \mathbf{R})$ and obtained some oscillation criteria, which improved and extended the results that have been established in [6-8].

Li et al. in [9] also discussed the oscillation of (1.2), where $\gamma>0$ is the quotient of odd positive integers, $f \in C(\mathbf{T} \times \mathbf{R}, \mathbf{R})$ is assumed to satisfy $u f(t, u)>0$ for $u \neq 0$ and there exists a positive rd-continuous function $p$ on $\mathbf{T}$ such that $\frac{f(t, u)}{u^{\gamma}} \geq p(t)$ for $u \neq 0$. They established some new sufficient conditions for the oscillation of (1.2).

Wang and $\mathrm{Xu}$ in [10] extended the Hille and Nehari oscillation theorems to the thirdorder dynamic equation

$$
\left(r_{2}(t)\left(\left(r_{1}(t) x^{\Delta}(t)\right)^{\Delta}\right)^{\gamma}\right)^{\Delta}+q(t) f(x(t))=0
$$

on a time scale $\mathbf{T}$, where $\gamma \geq 1$ is a ratio of odd positive integers and the functions $r_{i}(t)$ $(i=1,2), q(t)$ are positive real-valued rd-continuous functions defined on $\mathbf{T}$.

Erbe et al. in [11] were concerned with the oscillation of the third-order nonlinear functional dynamic equation

$$
\left(a(t)\left[\left(r(t) x^{\Delta}(t)\right)^{\Delta}\right]^{\gamma}\right)^{\Delta}+f(t, x(g(t)))=0
$$


on a time scale $\mathbf{T}$, where $\gamma$ is the quotient of odd positive integers, $a$ and $r$ are positive rdcontinuous functions on $\mathbf{T}$, and $g: \mathbf{T} \rightarrow \mathbf{T}$ satisfies $\lim _{t \rightarrow \infty} g(t)=\infty$ and $f \in C(\mathbf{T} \times \mathbf{R}, \mathbf{R})$. The authors obtained some new oscillation criteria and extended many known results for oscillation of third-order dynamic equations.

Qi and Yu in [12] obtained some oscillation criteria for the fourth-order nonlinear delay dynamic equation

$$
x^{\Delta^{4}}(t)+p(t) x^{\gamma}(\tau(t))=0
$$

on a time scale $\mathbf{T}$, where $\gamma$ is the ratio of odd positive integers, $p$ is a positive real-valued rd-continuous function defined on $\mathbf{T}, \tau \in C_{\mathrm{rd}}(\mathbf{T}, \mathbf{T}), \tau(t) \leq t$, and $\lim _{t \rightarrow \infty} \tau(t)=\infty$.

Grace $e t$ al. in [13] were concerned with the oscillation of the fourth-order nonlinear dynamic equation

$$
x^{\Delta^{4}}(t)+q(t) x^{\lambda}(t)=0
$$

on a time scale $\mathbf{T}$, where $\lambda$ is the ratio of odd positive integers, $q$ is a positive real-valued rd-continuous function defined on $\mathbf{T}$. They reduced the problem of the oscillation of all solutions of (1.3) to the problem of oscillation of two second-order dynamic equations and gave some conditions to ensure that all bounded solutions of (1.3) are oscillatory.

Grace et al. in [14] established some new criteria for the oscillation of the fourth-order nonlinear dynamic equation

$$
\left(a(t) x^{\Delta^{2}}(t)\right)^{\Delta^{2}}+f\left(t, x^{\sigma}(t)\right)=0, \quad t \geq t_{0}
$$

where $a$ is a positive real-valued rd-continuous function satisfying $\int_{t_{0}}^{\infty} \frac{\sigma(s)}{a(s)} \Delta s<\infty, f$ : $\left[t_{0}, \infty\right)_{\mathbf{T}} \times \mathbf{R} \rightarrow \mathbf{R}$ is continuous satisfying $\operatorname{sgn} f(t, x)=\operatorname{sgn} x$ and $f(t, x) \leq f(t, y)$ for $x \leq y$ and $t \geq t_{0}$. They also investigate the case of strongly superlinear and the case of strongly sublinear equations subject to various conditions.

Agarwal et al. in [15] were concerned with oscillatory behavior of a fourth-order halflinear delay dynamic equation with damping

$$
\left(r(t)\left(x^{\Delta^{3}}(t)\right)^{\gamma}\right)^{\Delta}+p(t)\left(x^{\Delta^{3}}(t)\right)^{\gamma}+q(t) x^{\gamma}(\tau(t))=0
$$

on a time scale $\mathbf{T}$ with $\sup \mathbf{T}=\infty$, where $\lambda$ is the ratio of odd positive integers, $r, p, q$ are positive real-valued rd-continuous functions defined on $\mathbf{T}, r(t)-\mu(t) p(t) \neq 0, \tau \in$ $C_{\mathrm{rd}}(\mathbf{T}, \mathbf{T}), \tau(t) \leq t$ and $\tau(t) \rightarrow \infty$ as $t \rightarrow \infty$. They established some new oscillation criteria of (1.4).

Zhang et al. in [16] concerned with the oscillation of a fourth-order nonlinear dynamic equation

$$
\left(p(t) x^{\Delta^{3}}(t)\right)^{\Delta}+q(t) f(x(\sigma(t)))=0
$$

on an arbitrary time scale $\mathbf{T}$ with $\sup \mathbf{T}=\infty$, where $p, q \in C_{\mathrm{rd}}(\mathbf{T},(0, \infty))$ with $\int_{t_{0}}^{\infty} \frac{1}{p(s)} \Delta s<$ $\infty$ and there exists a positive constant $L$ such that $\frac{f(y)}{y} \geq L$ for all $y \neq 0$, they gave a new oscillation result of (1.5). 
In [17], Sun et al. studied the following higher order dynamic equation:

$$
S_{n}^{\Delta}(t, x(t))+p(t) x^{\beta}(t)=0
$$

and established some new oscillation criteria.

For much research concerning the oscillation and nonoscillation of solutions of higher order dynamic equations on time scales, please refer to the literature [18-27].

\section{Some lemmas}

In order to obtain the main results of this paper, we need the following lemmas.

Lemma $2.1[28]$ Assume that

$$
\int_{t_{0}}^{\infty}\left[\frac{1}{a_{n}(s)}\right]^{\frac{1}{\alpha}} \Delta s=\int_{t_{0}}^{\infty} \frac{\Delta s}{a_{i}(s)}=\infty \text { for all } 1 \leq i \leq n-1,
$$

and integer $m \in[1, n]$. Then:

(1) $\liminf _{t \rightarrow \infty} S_{m}(t, x(t))>0$ implies $\lim _{t \rightarrow \infty} S_{i}(t, x(t))=\infty$ for $i \in[0, m-1]$.

(2) $\limsup _{t \rightarrow \infty} S_{m}(t, x(t))<0$ implies $\lim _{t \rightarrow \infty} S_{i}(t, x(t))=-\infty$ for $i \in[0, m-1]$.

Lemma 2.2 [28] Assume that (2.1) holds. If $S_{n}^{\Delta}(t, x(t))<0$ and $x(t)>0$ for $t \geq t_{0}$, then there exists an integer $m \in[0, n]$ such that:

(1) $m+n$ is even.

(2) $(-1)^{m+i} S_{i}(t, x(t))>0$ for $t \geq t_{0}$ and $i \in[m, n]$.

(3) If $m \geq 1$, then there exists $T \geq t_{0}$ such that $S_{i}(t, x(t))>0$ for $t \geq T$ and $i \in[1, m-1]$.

Lemma 2.3 [28] Assume that (2.1) holds. Furthermore, suppose that

$$
\int_{t_{0}}^{\infty} \frac{1}{a_{n-1}(u)}\left\{\int_{u}^{\infty}\left[\frac{1}{a_{n}(s)} \int_{s}^{\infty} q(v) \Delta v\right]^{\frac{1}{\alpha}} \Delta s\right\} \Delta u=\infty
$$

If $x$ is an eventually positive solution of (1.1), then there exists sufficiently large $T \geq t_{0}$ such that:

(1) $S_{n}^{\Delta}(t, x(t))<0$ for $t \geq T$.

(2) Either $\lim _{t \rightarrow \infty} x(t)=0$ or $S_{i}(t, x(t))>0$ for $t \geq T$ and $0 \leq i \leq n$.

Lemma 2.4 [28] Assume that $x$ is an eventually positive solution of (1.1). If there exists $T \geq t_{0}$ such that:

(1) $S_{n}^{\Delta}(t, x(t))<0$ for $t \geq T$.

(2) $S_{i}(t, x(t))>0$ for $t \geq T$ and $0 \leq i \leq n$.

Then

$$
S_{i}(t, x(t)) \geq S_{n}^{\frac{1}{\alpha}}(t, x(t)) B_{i+1}(t, T) \quad \text { for } 0 \leq i \leq n-1 \text { and } t \geq T
$$

and there exist $T_{1}>T$ and a constant $c>0$ such that

$$
x(t) \leq c B_{1}(t, T) \quad \text { for } t \geq T_{1},
$$


where

$$
B_{i}(t, T)= \begin{cases}\int_{T}^{t}\left[\frac{1}{a_{n}(s)}\right]^{\frac{1}{\alpha}} \Delta s, & \text { if } i=n, \\ \int_{T}^{t} \frac{B_{i+1}(s, T)}{a_{i}(s)} \Delta s, & \text { if } 1 \leq i \leq n-1 .\end{cases}
$$

Lemma 2.5 [3] Let $f: \mathbf{R} \rightarrow \mathbf{R}$ be continuously differentiable and suppose that $g: \mathbf{T} \rightarrow \mathbf{R}$ is delta differentiable. Then $f \circ g$ is delta differentiable and

$$
(f \circ g)^{\Delta}(t)=g^{\Delta}(t) \int_{0}^{1} f^{\prime}\left(h g(t)+(1-h) g^{\sigma}(t)\right) d h .
$$

Lemma 2.6 [17] If $A, B$ are nonnegative numbers and $\lambda>1$, then

$$
A^{\lambda}-\lambda A B^{\lambda-1}+(\lambda-1) B^{\lambda} \geq 0 .
$$

Lemma 2.7 [29] Assume that $U, V$ are constants and $\gamma \geq 1$ is the quotient of odd positive integers. Then

$$
(U-V)^{1+\frac{1}{\gamma}} \geq U^{1+\frac{1}{\gamma}}+\frac{1}{\gamma} V^{1+\frac{1}{\gamma}}-\left(1+\frac{1}{\gamma} V^{\frac{1}{\gamma}} U\right)
$$

\section{Main results}

Throughout this paper, we assume that:

(1) $\tau \circ \sigma=\sigma \circ \tau$, where the forward jump operator $\sigma: \mathbf{T} \rightarrow \mathbf{T}$ by $\sigma(t)=\inf \{s \in \mathbf{T}: s>t\}$.

(2) $\Phi: \mathbf{T} \rightarrow(0, \infty)$ and $\phi: \mathbf{T} \rightarrow[0, \infty)$ such that $\Phi(t)$ and $a(t) \phi(t)$ are differentiable.

Write

$$
\begin{aligned}
& h_{1}(t, T)=\frac{B_{2}(t, T)}{a_{1}(t)}, \\
& h_{2}(t, T)=h_{1}(t, T) B_{1}^{\alpha-1}(\sigma(t), T)=h_{1}(t, T)\left(B_{1}^{\alpha-1}(t, T)\right)^{\sigma}, \\
& \delta_{1}\left(t, T, c_{1}, c_{2}\right)= \begin{cases}c_{1}, c_{1} \text { are any positive constant, } \\
1, & \text { if } \alpha<\beta, \\
c_{2} B_{1}^{\beta-\alpha}(\sigma(t), T), c_{2} \text { are any positive constant, } & \text { if } \alpha=\beta,\end{cases} \\
& \delta_{2}\left(t, T, c_{1}, c_{2}\right)= \begin{cases}c_{1}, c_{1} \text { are any positive constant, } \\
1, & \text { if } \alpha<\beta, \\
c_{2} B_{1}^{\frac{\beta}{\alpha}-1}(\sigma(t), T), c_{2} \text { are any positive constant, }, & \text { if } \alpha>\beta,\end{cases} \\
& g_{1}\left(t, T, c_{1}, c_{2}\right)= \\
& \Phi_{2}^{\Delta}(t)+2 \beta \Phi(t) \tau^{\Delta}(t) h_{2}(\tau(t), T) \delta_{1}\left(\tau(t), T, c_{1}, c_{2}\right)\left(a_{n}(t) \phi(t)\right)^{\sigma},
\end{aligned}
$$




$$
\begin{gathered}
\times \delta_{2}\left(\tau(t), T, c_{1}, c_{2}\right)\left(\left(a_{n}(t) \phi(t)\right)^{\sigma}\right)^{1+\frac{1}{\alpha}}, \\
g_{+}=\max \{0, g\}, \quad g_{-}=\min \{0,-g\}, \\
X(t)=\left\{a_{n}(t)\left[\frac{\left[S_{n-1}^{\Delta}(t, x(t))\right]^{\alpha}}{x^{\beta}(\tau(t))}+\phi(t)\right]\right\} .
\end{gathered}
$$

Theorem 3.1 Suppose that (2.1) and (2.2) hold. If there exist differentiable functions $\Phi: \mathbf{T} \rightarrow(0, \infty)$ and $\phi: \mathbf{T} \rightarrow[0, \infty)$ with $a_{n}(t) \phi(t)$ being differentiable such that for all sufficiently large $T \in\left[t_{0}, \infty\right)_{\mathrm{T}}$ and for any positive constants $c_{1}, c_{2}$, there is a $T_{1}>T$ with $\tau\left(T_{1}\right)>T$ such that

$$
\limsup _{t \rightarrow \infty} \int_{T_{1}}^{t}\left[G_{1}\left(s, T, c_{1}, c_{2}\right)-\frac{g_{1}^{2}\left(s, T, c_{1}, c_{2}\right)}{4 \beta \Phi(s) \tau^{\Delta}(s) h_{2}(\tau(s), T) \delta_{1}\left(\tau(s), T, c_{1}, c_{2}\right)}\right] \Delta s=\infty,
$$

then every solution of (1.1) is either oscillatory or tends to 0.

Proof Assume that (1.1) has a nonoscillatory solution $x$ on $\left[t_{0}, \infty\right)_{\mathrm{T}}$. Then, without loss of generality, there is a sufficiently large $t_{1} \geq t_{0}$ such that $x(t)>0$ for $t \geq t_{1}$. Therefore from Lemma 2.3, we know that there exists sufficiently large $T \geq t_{1}$ such that:

(1) $S_{n}^{\Delta}(t, x(t))<0$ for $t \geq T$.

(2) Either $\lim _{t \rightarrow \infty} x(t)=0$ or $S_{i}(t, x(t))>0$ for $t \geq T$ and $0 \leq i \leq n$.

Let $S_{i}(t, x(t))>0$ for $t \geq T$ and $0 \leq i \leq n$. Consider

$$
w(t)=\Phi(t) a_{n}(t)\left[\frac{\left(S_{n-1}^{\Delta}(t, x(t))\right)^{\alpha}}{x^{\beta}(\tau(t))}+\phi(t)\right] .
$$

Then $X(t)=w^{\sigma}(t) / \Phi^{\sigma}(t)$ and $w(t)>0$ for $t \geq T$.

By the product rule and the quotient rule

$$
\begin{aligned}
w^{\Delta}(t)= & \frac{\Phi(t)}{x^{\beta}(\tau(t))} S_{n}^{\Delta}(t, x(t))+\left[\frac{\Phi(t)}{x^{\beta}(\tau(t))}\right]^{\Delta} S_{n}^{\sigma}(t, x(t)) \\
& +\Phi(t)\left[a_{n}(t) \phi(t)\right]^{\Delta}+\Phi^{\Delta}(t)\left[a_{n}(t) \phi(t)\right]^{\sigma} .
\end{aligned}
$$

Since $g(t, x(\tau(t))) / x^{\beta}(\tau(t)) \geq q(t)(x(t)>0)$, we get

$$
\begin{aligned}
w^{\Delta}(t) \leq & -\Phi(t) q(t)+\Phi(t)\left[a_{n}(t) \phi(t)\right]^{\Delta}+\Phi^{\Delta}(t) X(t) \\
& -\Phi(t)\left[\frac{\left(x^{\beta}(\tau(t))\right)^{\Delta}}{x^{\beta}(\tau(t))}\right]\left[\frac{S_{n}(t, x(t))}{x^{\beta}(\tau(t))}\right]^{\sigma} .
\end{aligned}
$$

Using the fact that $x$ and $\tau$ are differentiable functions and $\tau \circ \sigma=\sigma \circ \tau$, we see that $x \circ \tau$ is a differentiable function and $(x(\tau(t)))^{\Delta}=x^{\Delta}(\tau(t)) \tau^{\Delta}(t)$. Note $\beta \geq 1$. From Lemma 2.5, we get

$$
\left(x^{\beta}(\tau(t))\right)^{\Delta} \geq \beta x^{\beta-1}(\tau(t)) x^{\Delta}(\tau(t)) \tau^{\Delta}(t),
$$

which implies

$$
\begin{aligned}
w^{\Delta}(t) \leq & -\Phi(t) q(t)+\Phi(t)\left[a_{n}(t) \phi(t)\right]^{\Delta}+\Phi^{\Delta}(t) X(t) \\
& -\beta \Phi(t) \tau^{\Delta}(t) \frac{x^{\Delta}(\tau(t))}{x(\tau(t))}\left[\frac{S_{n}(t, x(t))}{x^{\beta}(\tau(t))}\right]^{\sigma} .
\end{aligned}
$$


We choose $t_{2} \geq T$ such that $\tau(t)>T$ for $t \geq t_{2}$. Then, from (2.3) and the fact that $S_{n}^{\Delta}(t$, $x(t))<0$ for $t \geq T$, we get

$$
\begin{aligned}
x^{\Delta}(\tau(t)) & \geq S_{n}^{\frac{1}{\alpha}}(\tau(t), x(t)) \frac{B_{2}(\tau(t), T)}{a_{1}(\tau(t))} \\
& \geq\left[S_{n}^{\frac{1}{\alpha}}(t, x(t))\right]^{\sigma} h_{1}(\tau(t), T) \\
& =h_{1}(\tau(t), T)\left(a_{n}^{\frac{1}{\alpha}}(t) S_{n-1}^{\Delta}(t, x(t))\right)^{\sigma} \\
& =h_{1}(\tau(t), T)\left[\frac{S_{n}(t, x(t))}{x^{\beta}(\tau(t))}\right]^{\sigma}\left[\frac{x^{\beta}(\tau(t))}{S_{n}^{\frac{\alpha-1}{\alpha}}(\tau(t, x(t)))}\right]^{\sigma} .
\end{aligned}
$$

From (2.3), we have

$$
x(t) \geq S_{n}^{\frac{1}{\alpha}}(t, x(t)) B_{1}(t, T) .
$$

Thus

$$
S_{n}^{\frac{\alpha-1}{\alpha}}(\tau(t), x(t)) \leq \frac{x^{\alpha-1}(\tau(t))}{B_{1}^{\alpha-1}(\tau(t), T)},
$$

which combines with (3.8) to imply

$$
x^{\Delta}(\tau(t)) \geq h_{2}(\tau(t), T)\left[\frac{\left(S_{n}(t, x(t))\right)}{x^{\beta}(\tau(t))}\right]^{\sigma}\left[\frac{x^{\beta}(\tau(t))}{x^{\alpha-1}(\tau(t))}\right]^{\sigma} .
$$

Combining (3.9) with (3.6) and from $\frac{x^{\sigma}(\tau(t))}{x(\tau(t))} \geq 1$, we obtain that

$$
\begin{aligned}
w^{\Delta}(t) \leq & -\Phi(t) q(t)+\Phi(t)\left[a_{n}(t) \phi(t)\right]^{\Delta}+\Phi^{\Delta}(t) X(t) \\
& -\beta \Phi(t) \tau^{\Delta}(t) h_{2}(\tau(t), T)\left[\frac{x^{\beta}(\tau(t))}{x^{\alpha}(\tau(t))}\right]^{\sigma}\left\{\left[\frac{S_{n}(t, x(t))}{x^{\beta}(\tau(t))}\right]^{\sigma}\right\}^{2} .
\end{aligned}
$$

Now we consider the following three cases.

Case (i). If $\alpha<\beta$, then $x^{\Delta}(t)>0$ for $t \geq T$ and $x(t) \geq x(T)=b_{1}>0$. Thus

$$
\left(x^{\beta-\alpha}(\tau(t))\right)^{\sigma} \geq b_{1}^{\beta-\alpha}=c_{1}>0 \quad \text { for } t \geq t_{2} .
$$

Case (ii). If $\alpha=\beta$, then

$$
\left(x^{\beta-\alpha}(\tau(t))\right)^{\sigma}=1 \quad \text { for } t \geq t_{2} .
$$

Case (iii). If $\alpha>\beta$, then from (2.4) we get that there exist $t_{3}>t_{2}$ and a constant $c>0$ such that

$$
x(t) \leq c B_{1}(t, T) \text { for } t \geq t_{3} .
$$

Thus

$$
\left(x^{\beta-\alpha}(\tau(t))\right)^{\sigma} \geq c_{2}\left(B_{1}^{\beta-\alpha}(\tau(t), T)\right)^{\sigma} \quad \text { for } t \geq t_{3},
$$

where $c_{2}=c^{\beta-\alpha}>0$. 
We obtain from the above that

$$
\left(\frac{x^{\beta}(\tau(t))}{x^{\alpha}(\tau(t))}\right)^{\sigma} \geq \delta_{1}\left(\tau(t), T, c_{1}, c_{2}\right)
$$

Thus

$$
\begin{aligned}
w^{\Delta}(t) \leq & -\Phi(t) q(t)+\Phi(t)\left[a_{n}(t) \phi(t)\right]^{\Delta}+\Phi^{\Delta}(t) X(t) \\
& -\beta \Phi(t) \tau^{\Delta}(t) h_{2}(\tau(t), T) \delta_{1}\left(\tau(t), T, c_{1}, c_{2}\right)\left\{\left[\frac{S_{n}(t, x(t))}{x^{\beta}(\tau(t))}\right]^{\sigma}\right\}^{2} .
\end{aligned}
$$

Since

$$
\begin{aligned}
{\left[\left(\frac{S_{n}(t, x(t))}{x^{\beta}(\tau(t))}\right)^{\sigma}\right]^{2} } & =\left(X(t)-\left(a_{n}(t) \phi(t)\right)^{\sigma}\right)^{2} \\
& =X^{2}(t)-2\left(a_{n}(t) \phi(t)\right)^{\sigma} X(t)+\left(\left(a_{n}(t) \phi(t)\right)^{\sigma}\right)^{2}
\end{aligned}
$$

from (3.10) and (3.11) and the definitions of $G_{1}\left(t, T, c_{1}, c_{2}\right)$ and $g_{1}\left(t, T, c_{1}, c_{2}\right)$, we get

$$
\begin{aligned}
w^{\Delta}(t) \leq & -G_{1}\left(t, T, c_{1}, c_{2}\right)+g_{1}\left(t, T, c_{1}, c_{2}\right) X(t) \\
& -\beta \Phi(t) \tau^{\Delta}(t) h_{2}(\tau(t), T) \delta_{1}\left(\tau(t), T, c_{1}, c_{2}\right) X^{2}(t) .
\end{aligned}
$$

It is easy to check that

$$
w^{\Delta}(t) \leq-G_{1}\left(t, T, c_{1}, c_{2}\right)+\frac{g_{1}^{2}\left(t, T, c_{1}, c_{2}\right)}{4 \beta \Phi(t) \tau^{\Delta}(t) h_{2}(\tau(t), T) \delta_{1}\left(\tau(t), T, c_{1}, c_{2}\right)} .
$$

Integrating both sides of the above inequality from $t_{3}$ to $t$, we get

$$
\begin{aligned}
& \int_{t_{3}}^{t}\left[G_{1}\left(s, T, c_{1}, c_{2}\right)-\frac{g_{1}^{2}\left(s, T, c_{1}, c_{2}\right)}{4 \beta \Phi(s) \tau^{\Delta}(s) h_{2}(\tau(s), T) \delta_{1}\left(\tau(s), T, c_{1}, c_{2}\right)}\right] \Delta s \\
& \quad \leq w\left(t_{3}\right)-w(t) \leq w\left(t_{3}\right),
\end{aligned}
$$

which leads to a contradiction to (3.3). The proof is completed.

Theorem 3.2 Suppose that (2.1) and (2.2) hold. If there exist differentiable functions $\Phi: \mathbf{T} \rightarrow(0, \infty)$ and $\phi: \mathbf{T} \rightarrow[0, \infty)$ with $a_{n}(t) \phi(t)$ being differentiable such that for all sufficiently large $T \in\left[t_{0}, \infty\right)_{\mathrm{T}}$ and for any positive constants $c_{1}, c_{2}$, there is a $T_{1}>T$ with $\tau\left(T_{1}\right)>T$ such that

$$
\begin{aligned}
& \limsup _{t \rightarrow \infty} \int_{T_{1}}^{t}\left[G_{2}\left(s, T, c_{1}, c_{2}\right)-\frac{\alpha^{\alpha}\left(\left(g_{2}\left(s, T, c_{1}, c_{2}\right)\right)_{+}\right)^{1+\alpha}}{(1+\alpha)^{1+\alpha}\left(\beta \Phi(s) \tau^{\Delta}(s) h_{1}(\tau(s), T) \delta_{2}\left(\tau(s), T, c_{1}, c_{2}\right)\right)^{\alpha}}\right] \Delta s \\
& \quad=\infty
\end{aligned}
$$

then every solution of (1.1) is either oscillatory or tends to 0. 
Proof Assume that (1.1) has a nonoscillatory solution $x$ on $\left[t_{0}, \infty\right)_{\mathrm{T}}$. Then, without loss of generality, there is a sufficiently large $t_{1} \geq t_{0}$ such that $x(t)>0$ for $t \geq t_{1}$. Therefore from Lemma 2.3, we know that there exists sufficiently large $T \geq t_{1}$ such that:

(1) $S_{n}^{\Delta}(t, x(t))<0$ for $t \geq T$.

(2) Either $\lim _{t \rightarrow \infty} x(t)=0$ or $S_{i}(t, x(t))>0$ for $t \geq T$ and $0 \leq i \leq n$.

Let $S_{i}(t, x(t))>0$ for $t \geq T$ and $0 \leq i \leq n$. From (3.7), we get

$$
\begin{aligned}
x^{\Delta}(\tau(t)) & \geq\left[S_{n}^{\frac{1}{\alpha}}(t, x(t))\right]^{\sigma} h_{1}(\tau(t), T) \\
& =h_{1}(\tau(t), T)\left(x^{\frac{\beta}{\alpha}}(\tau(t))\right)^{\sigma}\left\{\left[\frac{S_{n}(t, x(t))}{x^{\beta}(\tau(t))}\right]^{\sigma}\right\}^{\frac{1}{\alpha}} .
\end{aligned}
$$

Define $w(t)$ as (3.4). Choosing $t_{2} \geq T$ such that $\tau(t)>T$ for $t \geq t_{2}$. Combining (3.14) with (3.6), we see that for $t \in\left[t_{2}, \infty\right)_{\mathrm{T}}$,

$$
\begin{aligned}
w^{\Delta}(t) \leq & -\Phi(t) q(t)+\Phi(t)\left[a_{n}(t) \phi(t)\right]^{\Delta}+\Phi^{\Delta}(t) X(t) \\
& -\beta \Phi(t) \tau^{\Delta}(t) h_{1}(\tau(t), T)\left\{\left[\frac{S_{n}(t, x(t))}{x^{\beta}(\tau(t))}\right]^{\sigma}\right\}^{1+\frac{1}{\alpha}} \frac{\left(x^{\frac{\beta}{\alpha}}(\tau(t))\right)^{\sigma}}{x(\tau(t))} .
\end{aligned}
$$

Note $x(\tau(t)) \leq(x(\tau(t)))^{\sigma}$ since $x^{\Delta}(t)>0$, we obtain

$$
\begin{aligned}
w^{\Delta}(t) \leq & -\Phi(t) q(t)+\Phi(t)\left[a_{n}(t) \phi(t)\right]^{\Delta}+\Phi^{\Delta}(t) X(t) \\
& -\beta \Phi(t) \tau^{\Delta}(t) h_{1}(\tau(t), T)\left(\left(\frac{S_{n}(t, x(t))}{x^{\beta}(\tau(t))}\right)^{\sigma}\right)^{1+\frac{1}{\alpha}}\left(x^{\frac{\beta-\alpha}{\alpha}}(\tau(t))\right)^{\sigma} .
\end{aligned}
$$

Now we consider the following three cases.

Case (i). If $\alpha<\beta$, then

$$
\left(x^{\frac{\beta-\alpha}{\alpha}}(\tau(t))\right)^{\sigma} \geq x^{\frac{\beta-\alpha}{\alpha}}(T)=c_{1}>0 \quad \text { for } t \geq t_{2}
$$

since $x^{\Delta}(t)>0$ for $t \geq T$.

Case (ii). If $\alpha=\beta$, then

$$
\left(x^{\frac{\beta-\alpha}{\alpha}}(\tau(t))\right)^{\sigma}=1 \quad \text { for } t \geq t_{2} .
$$

Case (iii). If $\alpha>\beta$, then we get from (2.4) that there exist $t_{3}>t_{2}$ and a constant $c>0$ such that

$$
x(\tau(t)) \leq c B_{1}(\tau(t), T) \text { for } t \geq t_{3} .
$$

Thus

$$
\left(x^{\frac{\beta-\alpha}{\alpha}}(\tau(t))\right)^{\sigma} \geq c_{2}\left(B_{1}^{\frac{\beta-\alpha}{\alpha}}(\tau(t), T)\right)^{\sigma} \quad \text { for } t \geq t_{3},
$$

where $c_{2}=c^{\frac{\beta-\alpha}{\alpha}}>0$.

We obtain from the above

$$
\left(x^{\frac{\beta-\alpha}{\alpha}}(\tau(t))\right)^{\sigma} \geq \delta_{2}\left(\tau(t), T, c_{1}, c_{2}\right) .
$$


Then

$$
\begin{aligned}
w^{\Delta}(t) \leq & -\Phi(t) q(t)+\Phi(t)\left[a_{n}(t) \phi(t)\right]^{\Delta}+\Phi^{\Delta}(t) X(t) \\
& -\beta \Phi(t) \tau^{\Delta}(t) h_{1}(\tau(t), T) \delta_{2}\left(\tau(t), T, c_{1}, c_{2}\right)\left\{\left[\frac{S_{n}(t, x(t))}{x^{\beta}(\tau(t))}\right]^{\sigma}\right\}^{1+\frac{1}{\alpha}} .
\end{aligned}
$$

From Lemma 2.7, we have

$$
\begin{aligned}
\left(\left(\frac{S_{n}(t, x(t))}{x^{\beta}(\tau(t))}\right)^{\sigma}\right)^{1+\frac{1}{\alpha}}= & \left(X(t)-\left(a_{n}(t) \phi(t)\right)^{\sigma}\right)^{1+\frac{1}{\alpha}} \\
\geq & X^{1+\frac{1}{\alpha}}(t)+\frac{1}{\alpha}\left(a_{n}^{\sigma}(t) \phi^{\sigma}(t)\right)^{1+\frac{1}{\alpha}} \\
& -\left(1+\frac{1}{\alpha}\right)\left(a_{n}^{\sigma}(t) \phi^{\sigma}(t)\right)^{\frac{1}{\alpha}} X(t) .
\end{aligned}
$$

Combining (3.16) with (3.15) and the definitions of $G_{2}\left(t, T, c_{1}, c_{2}\right)$ and $g_{2}\left(t, T, c_{1}, c_{2}\right)$, we get

$$
\begin{aligned}
w^{\Delta}(t) \leq & -G_{2}\left(t, T, c_{1}, c_{2}\right)+g_{2}\left(t, T, c_{1}, c_{2}\right) X(t) \\
& -\beta \Phi(t) \tau^{\Delta}(t) h_{1}(\tau(t), T) \delta_{2}\left(\tau(t), T, c_{1}, c_{2}\right) X^{1+\frac{1}{\alpha}}(t) \\
\leq & -G_{2}\left(t, T, c_{1}, c_{2}\right)+\left(g_{2}\left(t, T, c_{1}, c_{2}\right)\right)_{+} X(t) \\
& -\beta \Phi(t) \tau^{\Delta}(t) h_{1}(\tau(t), T) \delta_{2}\left(\tau(t), T, c_{1}, c_{2}\right) X^{1+\frac{1}{\alpha}}(t) .
\end{aligned}
$$

Let

$$
\begin{aligned}
& A^{1+\frac{1}{\alpha}}=\beta \Phi(t) \tau^{\Delta}(t) h_{1}(\tau(t), T) \delta_{2}\left(\tau(t), T, c_{1}, c_{2}\right) X^{1+\frac{1}{\alpha}}(t), \\
& B^{\frac{1}{\alpha}}=\frac{\alpha\left(g_{2}\left(t, T, c_{1}, c_{2}\right)\right)_{+}}{(1+\alpha)\left(\beta \Phi(t) \tau^{\Delta}(t) h_{1}(\tau(t), T) \delta_{2}\left(\tau(t), T, c_{1}, c_{2}\right)\right)^{\frac{\alpha}{\alpha+1}}} .
\end{aligned}
$$

We have from Lemma 2.6

$$
\begin{aligned}
& \left(g_{2}\left(t, T, c_{1}, c_{2}\right)\right)_{+} X(t)-\beta \Phi(t) \tau^{\Delta}(t) h_{1}(\tau(t), T) \delta_{2}\left(\tau(t), T, c_{1}, c_{2}\right) X^{1+\frac{1}{\alpha}}(t) \\
& \leq \frac{\alpha^{\alpha}\left(\left(g_{2}\left(t, T, c_{1}, c_{2}\right)\right)_{+}\right)^{1+\alpha}}{(1+\alpha)^{1+\alpha}\left(\beta \Phi(t) \tau^{\Delta}(t) h_{1}(\tau(t), T) \delta_{2}\left(\tau(t), T, c_{1}, c_{2}\right)\right)^{\alpha}} .
\end{aligned}
$$

Then

$$
w^{\Delta}(t) \leq-G_{2}\left(t, T, c_{1}, c_{2}\right)+\frac{\alpha^{\alpha}\left(\left(g_{2}\left(t, T, c_{1}, c_{2}\right)\right)_{+}\right)^{1+\alpha}}{(1+\alpha)^{1+\alpha}\left(\beta \Phi(t) \tau^{\Delta}(t) h_{1}(\tau(t), T) \delta_{2}\left(\tau(t), T, c_{1}, c_{2}\right)\right)^{\alpha}} .
$$

Integrating both sides of the above inequality from $t_{3}$ to $t$, we get

$$
\begin{aligned}
& \int_{t_{3}}^{t}\left[G_{2}\left(s, T, c_{1}, c_{2}\right)-\frac{\alpha^{\alpha}\left(\left(g_{2}\left(s, T, c_{1}, c_{2}\right)\right)_{+}\right)^{1+\alpha}}{(1+\alpha)^{1+\alpha}\left(\beta \Phi(s) \tau^{\Delta}(s) h_{1}(\tau(s), T) \delta_{2}\left(\tau(s), T, c_{1}, c_{2}\right)\right)^{\alpha}}\right] \Delta s \\
& \quad \leq w\left(t_{3}\right)-w(t) \leq w\left(t_{3}\right),
\end{aligned}
$$

which leads to a contradiction to (3.13). The proof is completed. 


\section{Further results}

For convenience, let $D=\left\{(t, s) \in \mathbf{T}^{2}: t \geq s \geq t_{0}, t, s \in\left[t_{0}, \infty\right)_{\mathbf{T}}\right\}$. For any function $G: \mathbf{T}^{2} \rightarrow$ $\mathbf{R}$, denote by $G^{\Delta_{s}}$ the partial derivative of $G(t, s)$ with respect to $s$. Define

$$
\Im^{*}=\left\{G \in C_{\mathrm{rd}}(D,[0, \infty)): G(s, s)=0, G(t, s)>0, G^{\Delta_{s}} \leq 0, t>s \geq t_{0}\right\} .
$$

Theorem 4.1 Suppose that (2.1) and (2.2) hold. If there exist functions $r, R \in \mathfrak{\Im}^{*}$ and differentiable functions $\Phi: \mathbf{T} \rightarrow(0, \infty)$ and $\phi: \mathbf{T} \rightarrow[0, \infty)$ with $a_{n}(t) \phi(t)$ being differentiable such that for all sufficiently large $T \in\left[t_{0}, \infty\right)_{\mathrm{T}}$ and for any positive constants $c_{1}, c_{2}$, there is a $T_{1}>T$ with $\tau\left(T_{1}\right)>T$ such that

$$
R^{\Delta_{s}}(t, s)+\frac{R(t, s) g_{1}\left(s, T, c_{1}, c_{2}\right)}{\Phi^{\sigma}(s)}=\frac{r(t, s)}{\Phi^{\sigma}(s)} R^{\frac{1}{2}}(t, s)
$$

and

$$
\begin{aligned}
& \limsup _{t \rightarrow \infty} \frac{1}{R\left(t, T_{1}\right)} \int_{T_{1}}^{t}\left[R(t, s) G_{1}\left(s, T, c_{1}, c_{2}\right)\right. \\
& \left.-\frac{r^{2}(t, s)}{4 \beta \Phi(s) \tau^{\Delta}(s) h_{2}(\tau(s), T) \delta_{1}\left(\tau(s), T, c_{1}, c_{2}\right)}\right] \Delta s=\infty,
\end{aligned}
$$

then every solution of (1.1) is either oscillatory or tends to 0.

Proof Assume that (1.1) has a nonoscillatory solution $x$ on $\left[t_{0}, \infty\right)_{\mathrm{T}}$. Then, without loss of generality, there is a sufficiently large $t_{1} \geq t_{0}$ such that $x(t)>0$ for $t \geq t_{1}$. Therefore from Lemma 2.3, we know that there exists sufficiently large $T \geq t_{1}$ such that:

(1) $S_{n}^{\Delta}(t, x(t))<0$ for $t \geq T$.

(2) Either $\lim _{t \rightarrow \infty} x(t)=0$ or $S_{i}(t, x(t))>0$ for $t \geq T$ and $0 \leq i \leq n$.

Let $S_{i}(t, x(t))>0$ for $t \geq T$ and $0 \leq i \leq n$. Define $w(t)$ as (3.4). Choosing $t_{2} \geq T$ such that (3.12) holds for $t \geq t_{2}$. Then for $t \in\left[t_{2}, \infty\right)_{\mathrm{T}}$

$$
\begin{aligned}
G_{1}\left(t, T, c_{1}, c_{2}\right) \\
\quad \leq-w^{\Delta}(t)+g_{1}\left(t, T, c_{1}, c_{2}\right) X(t) \\
\quad-\beta \Phi(t) \tau^{\Delta}(t) h_{2}(\tau(t), T) \delta_{1}\left(\tau(t), T, c_{1}, c_{2}\right) X^{2}(t) .
\end{aligned}
$$

In (4.3), replace $t$ by $s$ and multiply both sides by $R(t, s)$, integrate with respect to $s$ from $t_{2}$ to $t>t_{2}$, we have

$$
\begin{aligned}
\int_{t_{2}}^{t} R(t, s) G_{1}\left(s, T, c_{1}, c_{2}\right) \Delta s \\
\leq- \\
\quad \int_{t_{2}}^{t} R(t, s) w^{\Delta}(s) \Delta s+\int_{t_{2}}^{t} R(t, s) g_{1}\left(s, T, c_{1}, c_{2}\right) X(s) \Delta s \\
\quad-\int_{t_{2}}^{t} R(t, s) \beta \Phi(s) \tau^{\Delta}(s) h_{2}(\tau(s), T) \delta_{1}\left(\tau(s), T, c_{1}, c_{2}\right) X^{2}(s) \Delta s .
\end{aligned}
$$


Integrating by parts and using (4.1), we get

$$
\begin{aligned}
& \int_{t_{2}}^{t} R(t, s) G_{1}\left(s, T, c_{1}, c_{2}\right) \Delta s \\
& \quad \leq R\left(t, t_{2}\right) w\left(t_{2}\right)+\int_{t_{2}}^{t}\left[r(t, s) R^{\frac{1}{2}}(t, s) X(s)\right. \\
& \left.\quad-R(t, s) \beta \Phi(s) \tau^{\Delta}(s) h_{2}(\tau(s), T) \delta_{1}\left(\tau(s), T, c_{1}, c_{2}\right) X^{2}(s)\right] \Delta s .
\end{aligned}
$$

This implies

$$
\begin{aligned}
& \int_{t_{2}}^{t} R(t, s) G_{1}\left(s, T, c_{1}, c_{2}\right) \Delta s \\
& \quad \leq R\left(t, t_{2}\right) w\left(t_{2}\right)+\int_{t_{2}}^{t} \frac{r^{2}(t, s)}{4 \beta \Phi(s) \tau^{\Delta}(s) h_{2}(\tau(s), T) \delta_{1}\left(\tau(s), T, c_{1}, c_{2}\right)} \Delta s .
\end{aligned}
$$

Thus

$$
\frac{1}{R\left(t, t_{2}\right)} \int_{t_{2}}^{t}\left[R(t, s) G_{1}(s, T)-\frac{r^{2}(t, s)}{4 \beta \Phi(s) \tau^{\Delta}(s) h_{2}(\tau(s), T) \delta_{1}\left(\tau(s), T, c_{1}, c_{2}\right)}\right] \Delta s \leq w\left(t_{2}\right),
$$

which leads to a contradiction to (4.2). The proof is completed.

Theorem 4.2 Suppose that (2.1) and (2.2) hold. If there exist functions $r, R \in \mathfrak{\Im}^{*}$ and differentiable functions $\Phi: \mathbf{T} \rightarrow(0, \infty)$ and $\phi: \mathbf{T} \rightarrow[0, \infty)$ with $a_{n}(t) \phi(t)$ being differentiable such that for all sufficiently large $T \in\left[t_{0}, \infty\right)_{\mathrm{T}}$ and for any positive constants $c_{1}, c_{2}$, there is a $T_{1}>T$ with $\tau\left(T_{1}\right)>T$ such that

$$
R^{\Delta_{s}}(t, s)+\frac{R(t, s) g_{2}\left(s, T, c_{1}, c_{2}\right)}{\Phi^{\sigma}(s)}=\frac{r(t, s)}{\Phi^{\sigma}(s)} R^{\frac{\alpha}{1+\alpha}}(t, s)
$$

and

$$
\begin{aligned}
& \limsup _{t \rightarrow \infty} \frac{1}{R\left(t, T_{1}\right)} \int_{T_{1}}^{t}\left[R(t, s) G_{2}\left(s, T, c_{1}, c_{2}\right)\right. \\
& \left.-\frac{\alpha^{\alpha}\left(r^{2}(t, s)\right)^{1+\alpha}}{(1+\alpha)^{1+\alpha}\left(\beta \Phi(s) \tau^{\Delta}(s) h_{2}(\tau(s), T) \delta_{1}\left(\tau(s), T, c_{1}, c_{2}\right)\right)^{\alpha}}\right] \Delta s=\infty,
\end{aligned}
$$

then every solution of (1.1) is either oscillatory or tends to 0.

Proof Assume that (1.1) has a nonoscillatory solution $x$ on $\left[t_{0}, \infty\right)_{\mathrm{T}}$. Then, without loss of generality, there is a sufficiently large $t_{1} \geq t_{0}$ such that $x(t)>0$ for $t \geq t_{1}$. Therefore from Lemma 2.3, we know that there exists sufficiently large $T \geq t_{1}$ such that:

(1) $S_{n}^{\Delta}(t, x(t))<0$ for $t \geq T$.

(2) Either $\lim _{t \rightarrow \infty} x(t)=0$ or $S_{i}(t, x(t))>0$ for $t \geq T$ and $0 \leq i \leq n$.

Let $S_{i}(t, x(t))>0$ for $t \geq T$ and $0 \leq i \leq n$. Define $w(t)$ as (3.4). Choosing $t_{2} \geq T$ such that (3.17) holds for $t \geq t_{2}$. Then for $t \in\left[t_{2}, \infty\right)_{\mathrm{T}}$, we have

$$
\begin{aligned}
G_{2}\left(t, T, c_{1}, c_{2}\right) \leq & -w^{\Delta}(t)+\frac{g_{2}\left(t, T, c_{1}, c_{2}\right)}{\Phi^{\sigma}(t)} w^{\sigma}(t) \\
& -\beta \Phi(t) \tau^{\Delta}(t) h_{1}(\tau(t), T) \delta_{1}\left(\tau(t), T, c_{1}, c_{2}\right) X^{\left(1+\frac{1}{\alpha}\right)}(t) .
\end{aligned}
$$


In (4.6), replace $t$ by $s$ and multiply both sides by $R(t, s)$ and integrate with respect to $s$ from $t_{2}$ to $t>t_{2}$, it follows that

$$
\begin{aligned}
& \int_{t_{2}}^{t} R(t, s) G_{2}\left(s, T, c_{1}, c_{2}\right) \Delta s \\
& \leq-\int_{t_{2}}^{t} R(t, s) w^{\Delta}(s) \Delta s+\int_{t_{2}}^{t} R(t, s) \frac{g_{2}\left(s, T, c_{1}, c_{2}\right)}{\Phi^{\sigma}(s)} w^{\sigma}(s) \Delta s \\
& \quad-\int_{t_{2}}^{t} R(t, s) \beta \Phi(s) \tau^{\Delta}(s) h_{1}(\tau(s), T) \delta_{2}\left(\tau(s), T, c_{1}, c_{2}\right) X^{\left(1+\frac{1}{\alpha}\right)}(s) \Delta s .
\end{aligned}
$$

Integrating by parts and using (4.4), we get

$$
\begin{aligned}
& \int_{t_{2}}^{t} R(t, s) G_{2}\left(s, T, c_{1}, c_{2}\right) \Delta s \\
& \quad \leq R\left(t, t_{2}\right) w\left(t_{2}\right)+\int_{t_{2}}^{t}\left[r_{+}(t, s) R^{\frac{\alpha}{1+\alpha}}(t, s) X(s)\right. \\
& \left.\quad-\beta R(t, s) \Phi(s) \tau^{\Delta}(s) h_{1}(\tau(s), T) \delta_{2}\left(\tau(s), T, c_{1}, c_{2}\right) X^{\left(1+\frac{1}{\alpha}\right)}(s)\right] \Delta s
\end{aligned}
$$

Let

$$
\begin{aligned}
& A^{1+\frac{1}{\alpha}}=\beta R(t, s) \Phi(s) \tau^{\Delta}(s) h_{1}(\tau(s), T) \delta_{2}\left(\tau(s), T, c_{1}, c_{2}\right) X^{1+\frac{1}{\alpha}}(s), \\
& B^{\frac{1}{\alpha}}=\frac{\alpha r_{+}(t, s)}{(1+\alpha)\left(\beta \Phi(s) \tau^{\Delta}(s) h_{1}(\tau(s), T) \delta_{2}\left(\tau(s), T, c_{1}, c_{2}\right)\right)^{\frac{\alpha}{\alpha+1}}} .
\end{aligned}
$$

From Lemma 2.6, we have

$$
\begin{aligned}
& \int_{t_{2}}^{t}\left[r_{+}(t, s) R^{\frac{\alpha}{1+\alpha}}(t, s) X(s)-\beta R(t, s) \Phi(s) \tau^{\Delta}(s) h_{1}(\tau(s), T) \delta_{2}\left(\tau(s), T, c_{1}, c_{2}\right) X^{1+\frac{1}{\alpha}}(s)\right] \Delta s \\
& \leq \int_{t_{2}}^{t} \frac{\alpha^{\alpha}\left(r_{+}(t, s)\right)^{1+\alpha}}{(1+\alpha)^{1+\alpha}\left(\beta \Phi(s) \tau^{\Delta}(s) h_{1}(\tau(s), T) \delta_{2}\left(\tau(s), T, c_{1}, c_{2}\right)\right)^{\alpha}} \Delta s
\end{aligned}
$$

which implies

$$
\begin{aligned}
& \int_{t_{2}}^{t}\left[R(t, s) G_{2}\left(s, T, c_{1}, c_{2}\right)\right. \\
& \left.\quad-\frac{\alpha^{\alpha}\left(r_{+}(t, s)\right)^{1+\alpha}}{(1+\alpha)^{1+\alpha}\left(\beta \Phi(s) \tau^{\Delta}(s) h_{1}(\tau(s), T) \delta_{2}\left(\tau(s), T, c_{1}, c_{2}\right)\right)^{\alpha}}\right] \Delta s \leq R\left(t, t_{2}\right) w\left(t_{2}\right) .
\end{aligned}
$$

Then

$$
\begin{aligned}
& \frac{1}{R\left(t, t_{2}\right)} \int_{t_{2}}^{t}\left[R(t, s) G_{2}\left(s, T, c_{1}, c_{2}\right)\right. \\
& \left.\quad-\frac{\alpha^{\alpha}\left(r_{+}(t, s)\right)^{1+\alpha}}{(1+\alpha)^{1+\alpha}\left(\beta \Phi(s) \tau^{\Delta}(t) h_{1}(\tau(s), T) \delta_{2}\left(\tau(s), T, c_{1}, c_{2}\right)\right)^{\alpha}}\right] \Delta s \leq w\left(t_{2}\right),
\end{aligned}
$$

which leads to a contradiction to (4.2). The proof is completed. 


\section{Example}

In this section, we give an example to illustrate our main results.

Example 5.1 Consider the following higher order dynamic equation:

$$
S_{n}^{\Delta}(t, x(t))+\frac{\gamma}{t^{\beta+1}} x^{\beta+1}(\tau(t))=0
$$

on time scale $\mathbf{T}=\{0\} \cup\left\{1 / 2^{k}: k=1,2,3, \ldots\right\} \cup\left\{2^{k}: k=1,2,3, \ldots\right\}$, where $n \geq 2, S_{k}(t)(0 \leq$ $k \leq n)$ is as in (1.1) with $a_{n}(t)=t^{\alpha}, a_{n-1}(t)=\cdots=a_{1}(t)=1, q(t)=\frac{\gamma}{t^{\beta+1}}, \gamma>1 / 2^{1-\beta}, 0<\beta<1$, $t_{0}=4$, and $\tau(t)=t / 2$. Then $\tau^{\Delta}(t)=1 / 2$ and the forward jump operator $\sigma(t)=2 t$ satisfies $\sigma(\tau(t))=\tau(\sigma(t))$. Thus

$$
\begin{aligned}
& \int_{t_{0}}^{\infty}\left(\frac{1}{a_{n}(s)}\right)^{\frac{1}{\alpha}} \Delta s=\int_{t_{0}}^{\infty} \frac{\Delta s}{s}=\infty, \\
& \int_{t_{0}}^{\infty} \frac{\Delta s}{a_{i}(s)}=\int_{t_{0}}^{\infty} \Delta s=\infty, \\
& \int_{t_{0}}^{\infty} \frac{1}{a_{n-1}(t)}\left\{\int_{t}^{\infty}\left[\frac{1}{a_{n}(s)} \int_{s}^{\infty} q(u) \Delta u\right]^{\frac{1}{\alpha}} \Delta s\right\} \Delta t \\
& =\int_{t_{0}}^{\infty}\left\{\int_{t}^{\infty}\left[\frac{1}{s^{\alpha}} \int_{s}^{\infty} \frac{\gamma}{u^{\beta+1}} \Delta u\right]^{\frac{1}{\alpha}} \Delta s\right\} \Delta t \\
& \geq\left(\frac{\gamma}{\beta}\right)^{\frac{1}{\alpha}} \int_{t_{0}}^{\infty}\left\{\int _ { t } ^ { \infty } \left[\frac{1}{s^{\alpha}} \int_{s}^{\infty} \frac{\left(u^{\beta}\right)^{\Delta}}{\left.\left.u^{\beta}\left(u^{\beta}\right)^{\sigma} \Delta u\right]^{\frac{1}{\alpha}} \Delta s\right\} \Delta t}\right.\right. \\
& =\left(\frac{\gamma}{\beta}\right)^{\frac{1}{\alpha}} \int_{t_{0}}^{\infty}\left[\int_{t}^{\infty} \frac{\Delta s}{s s^{\frac{\beta}{\alpha}}}\right] \Delta t \geq\left(\frac{\gamma}{\beta}\right)^{\frac{1}{\alpha}} \int_{t_{0}}^{\infty}\left[\int_{t}^{\infty} \frac{\Delta s}{s s^{\sigma}}\right] \Delta t \\
& =\left(\frac{\gamma}{\beta}\right)^{\frac{1}{\alpha}} \int_{t_{0}}^{\infty} \frac{1}{t} \Delta t=\infty .
\end{aligned}
$$

Therefore (2.1) and (2.2) hold. Note that

$$
\lim _{t \rightarrow \infty} B_{n}(t, T)=\lim _{t \rightarrow \infty} \int_{T}^{t}\left[\frac{1}{a_{n}(s)}\right]^{\frac{1}{\alpha}} \Delta s=\lim _{t \rightarrow \infty} \int_{T}^{t} \frac{1}{s} \Delta s=\infty .
$$

It is easy to check that

$$
\lim _{t \rightarrow \infty} B_{2}(t, T)=\lim _{t \rightarrow \infty} B_{1}(\sigma(t), T)=\infty
$$

and

$$
\lim _{t \rightarrow \infty} h_{2}(t, T)=\lim _{t \rightarrow \infty} h_{1}(\sigma(t), T)=\infty .
$$

Then for any positive constants $c_{1}, c_{2}$, there is a sufficiently large $t_{1}$ such that $\left(B_{1}(\tau(t), T)\right)^{\sigma}>$ 1 for $t \geq t_{1}, h_{1}(\tau(t), T) \geq 1 / c_{2}$, and $h_{2}(\tau(t), T) \geq \max \left\{1,1 / c_{1}\right\}$ for $t \geq t_{1}$,

$$
h_{2}(\tau(t), T) \delta_{1}\left(\tau(t), T, c_{1}, c_{2}\right)= \begin{cases}c_{1} h_{2}(\tau(t), T) \geq 1, & \text { if } \alpha<\beta+1, \\ h_{2}(\tau(t), T) \geq 1, & \text { if } \alpha=\beta+1, \\ c_{2} h_{1}(\tau(t), T)\left(B_{1}^{\beta}(\tau(t), T)\right)^{\sigma} \geq 1, & \text { if } \alpha>\beta+1 .\end{cases}
$$


Choosing $\phi(t)=0$ and $\Phi(t)=t$. Then $g_{1}\left(t, T, c_{1}, c_{2}\right)=\Phi^{\Delta}(t)=1$ and $G_{1}\left(t, T, c_{1}, c_{2}\right)=$ $\Phi(t) q(t)=\frac{\gamma}{t^{\beta}}$. Thus

$$
\begin{aligned}
& \limsup _{t \rightarrow \infty} \int_{T_{1}}^{t}\left[G_{1}\left(s, T, c_{1}, c_{2}\right)-\frac{g_{1}^{2}\left(s, T, c_{1}, c_{2}\right)}{4(\beta+1) \Phi(s) \tau^{\Delta}(s) h_{2}(\tau(s), T) \delta_{1}\left(\tau(s), T, c_{1}, c_{2}\right)}\right] \Delta s \\
& \geq \limsup _{t \rightarrow \infty} \int_{T_{1}}^{t}\left(\frac{\gamma}{s^{\beta}}-\frac{1}{2(\beta+1)} \frac{1}{s}\right) \Delta s \geq \limsup _{t \rightarrow \infty} \int_{T_{1}}^{t} \frac{1}{2 s} \Delta s=\infty .
\end{aligned}
$$

The conditions of Theorem 3.1 are satisfied. Then every solution of (5.1) is either oscillatory or tends to 0 .

Remark 5.1 If $\beta=0$, then the conditions of Theorem 3.1 are also satisfied and every solution of (5.1) is also either oscillatory or tends to 0 .

Remark 5.2 In Example 5.1, let $R(t, s)=r(t, s)=1$ for $t>s \geq 4$ and $R(t, t)=r(t, t)=0$ for $t \geq 4$. Then the conditions of Theorem 4.1 are satisfied. It also follows from Theorem 4.1 that every solution of (5.1) is either oscillatory or tends to 0 .

\section{Competing interests}

The authors declare that they have no competing interests.

\section{Authors' contributions}

All authors contributed equally and significantly in writing this article. All authors read and approved the final manuscript.

\section{Author details}

${ }^{1}$ Guangxi Colleges and Universities Key Laboratory of Mathematics and Its Applications, Nanning, Guangxi 530004,

P.R. China. ${ }^{2}$ College of Mathematics and Information Science, Guangxi University, Nanning, Guangxi 530004, P.R. China.

${ }^{3}$ College of Electrical Engineering, Guangxi University, Nanning, Guangxi 530004, P.R. China.

\section{Acknowledgements}

This project is supported by NNSF of China (11461003) and NSF of Guangxi (2012GXNSFDA276040).

Received: 27 September 2014 Accepted: 9 December 2014 Published: 30 Dec 2014

\section{References}

1. Hilger, S: Analysis on measure chains - a unified approach to continuous and discrete calculus. Results Math. 18 18-56 (1990)

2. Kac, V, Chueng, P: Quantum Calculus. Universitext. Springer, New York (2002)

3. Bohner, M, Peterson, A: Dynamic Equations on Time Scales: An Introduction with Applications. Birkhäuser, Boston (2001)

4. Bohner, M, Peterson, A: Advances in Dynamic Equations on Time Scales. Birkhäuser, Boston (2003)

5. Hassan, TS: Oscillation of third order nonlinear delay dynamic equations on time scales. Math. Comput. Model. 49 1573-1586 (2009)

6. Erbe, L, Peterson, A, Saker, SH: Asymptotic behavior of solutions of a third-order nonlinear dynamic equation on time scales. J. Comput. Appl. Math. 181, 92-102 (2005)

7. Erbe, L, Peterson, A, Saker, SH: Oscillation and asymptotic behavior a third-order nonlinear dynamic equation. Can. Appl. Math. Q. 14, 129-147 (2006)

8. Erbe, L, Peterson, A, Saker, SH: Hille and Nehari type criteria for third-order dynamic equations. J. Math. Anal. Appl. 329, 112-131 (2007)

9. Li, T, Han, Z, Sun, S, Zhao, Y: Oscillation results for third order nonlinear delay dynamic equations on time scales. Bull. Malays. Math. Soc. 34, 639-648 (2011)

10. Wang, $Y, X u, Z$ : Asymptotic properties of solutions of certain third-order dynamic equations. J. Comput. Appl. Math. 236, 2354-2366 (2012)

11. Erbe, L, Hassan, TS, Peterson, A: Oscillation of third order nonlinear functional dynamic equations on time scales. Differ. Equ. Dyn. Syst. 18, 199-227 (2008)

12. Qi, Y, Yu, J: Oscillation criteria for fourth-order nonlinear delay dynamic equations. Electron. J. Differ. Equ. 2013,79 (2013)

13. Grace, SR, Bohner, M, Sun, S: Oscillation of fourth-order dynamic equations. Hacet. J. Math. Stat. 39, 545-553 (2010)

14. Grace, SR, Sun, S, Wang, Y: On the oscillation of fourth order strongly superlinear and strongly sublinear dynamic equations. J. Appl. Math. Comput. 44, 119-132 (2014) 
15. Agarwal, RP, Bohner, M, Li, T, Zhang, C: Oscillation theorems for fourth-order half-linear delay dynamic equations with damping. Mediterr. J. Math. 11, 463-475 (2014)

16. Zhang, C, Li, T, Agarwal, RP, Bohner, M: Oscillation results for fourth-order nonlinear dynamic equations. Appl. Math. Lett. 25, 2058-2065 (2012)

17. Sun, $T, H e, Q, X i, H, Y u, W$ : Oscillation for higher order dynamic equations on time scales. Abstr. Appl. Anal. 2013, Article ID 268721 (2013)

18. Agarwal, RP, Bohner, M, Li, T, Zhang, C: Oscillation criteria for second-order dynamic equations on time scales. Appl. Math. Lett. 31, 34-40 (2014)

19. Grace, SR, Hassan, TS: Oscillation criteria for higher order nonlinear dynamic equations. Math. Nachr. 287, 1659-1673 (2014)

20. Graef, JR, Panigrahi, S, Reddy, PR: On oscillatory and asymptotic behavior of fourth order nonlinear neutral delay dynamic equations with positive and negative coefficients. Math. Slovaca 64, 347-366 (2014)

21. Li, L, Han, M: Some new dynamic Opial type inequalities and applications for second order integro-differential dynamic equations on time scales. Appl. Math. Comput. 232, 542-547 (2014)

22. Sun, $T, X i, H, P e n, X, Y u, W$ : Nonoscillatory solutions for higher-order neutral dynamic equations on time scales. Abstr. Appl. Anal. 2010, Article ID 428963 (2010)

23. Sun, T, Xi, H, Yu, W: Asymptotic behaviors of higher order nonlinear dynamic equations on time scales. J. Appl. Math. Comput. 37, 177-192 (2011)

24. Sun, $T, X i, H$, Pen, $X$ : Asymptotic behavior of solutions of higher-order dynamic equations on time scales. Adv. Differ. Equ. 2011, Article ID 237219 (2011)

25. Wu, X, Sun, T, Xi, H, Chen, C: Kamenev-type oscillation criteria for higher-order nonlinear dynamic equations on time scales. Adv. Differ. Equ. 2013, 248 (2013)

26. $\mathrm{Wu}, \mathrm{X}, \mathrm{Sun}, \mathrm{T}, \mathrm{Xi}, \mathrm{H}, \mathrm{Chen}, \mathrm{C}$ : Oscillation criteria for fourth-order nonlinear dynamic equations on time scales. Abstr. Appl. Anal. 2013, Article ID 740568 (2013)

27. $\mathrm{Wu}, \mathrm{X}, \mathrm{Sun}, \mathrm{T}$ : Oscillation criteria for higher order nonlinear delay dynamic equations on time scales. Math. Slovaca (2014, in press)

28. Sun, $T, Y u, W, X i, H$ : Oscillatory behavior and comparison for higher order nonlinear dynamic equations on time scales. J. Appl. Math. Inform. 30, 289-304 (2012)

29. Gera, M, Graef, JR, Gregus, M: On oscillatory and asymptotic properties of solutions of certain nonlinear third order differential equations. Nonlinear Anal., Theory Methods Appl. 32, 417-425 (1998)

10.1186/1687-1847-2014-328

Cite this article as: Sun et al.: New oscillation criteria for higher order delay dynamic equations on time scales. Advances in Difference Equations 2014, 2014:328

\section{Submit your manuscript to a SpringerOpen ${ }^{\circ}$ journal and benefit from:}

- Convenient online submission

Rigorous peer review

- Immediate publication on acceptance

- Open access: articles freely available online

- High visibility within the field

- Retaining the copyright to your article 